\title{
Chromospheric plasma and the Farley-Buneman instability in solar magnetic regions
}

\author{
J. M. Fontenla \\ Laboratory for Atmospheric and Space Physics (LASP), University of Colorado, Boulder, CO, 80309, USA \\ e-mail: fontenla@lasp.colorado.edu \\ Received 21 June 2005 / Accepted 26 July 2005

\section{ABSTRACT} \\ We study the plasma parameters in recent models of the observed magnetic features in the solar atmosphere and find that electrons are strongly \\ magnetized in the chromosphere but protons are unmagnetized up to the transition region. Considering the magnetization and the classical \\ Pedersen conductivity we find that magnetic diffusion is too small for effectively affecting propagating MHD waves of periods of a few \\ minutes. However, the chromospheric-plasma parameters suggest a scenario in which upward-propagating fast-mode $\mathrm{MHD}$ waves of $\mathrm{mHz}$ \\ frequencies would trigger the Farley-Buneman plasma instability at chromospheric layers where horizontal magnetic fields are present. We \\ show that, because of the collisions between charged particles and neutral $\mathrm{H}$ atoms, the conditions in the chromosphere meet the instability \\ criteria if the MHD wave velocity amplitude is lower but near the adiabatic sound speed. The instability growth is much faster than the wave \\ frequency and the instability would quickly saturate. The electrostatic plasma waves resulting from the instability are expected to produce \\ anomalous resistivity and wave energy dissipation that would heat the chromosphere as well as absorb the p-modes in magnetic regions.
}

Key words. Sun: chromosphere - Sun: faculae, plages - Sun: magnetic fields

\section{Introduction}

Magnetic regions on the solar surface display a pattern of sustained chromospheric heating that is yet unexplained. Such heating is well correlated with the magnetic fields out of sunspots. According to the latest models the amount of chromospheric heating increases with magnetic field but appears reduced in large sunspots (Fontenla et al. 2005). We study here the conditions of the plasma in the mid- and upperchromosphere where the magnetic heating manifests in strong chromospheric emissions such as those in the UV Lyman continuum and the peaks of the Ly $\alpha$ line, and enhanced line cores such as the $\mathrm{Ca}$ II $\mathrm{H}$ and $\mathrm{K}$ and the $\mathrm{Mg}$ II $\mathrm{h}$ and $\mathrm{k}$ lines (e.g. Lemaire et al. 1981). These emissions show an almost steady heating of the mid and upper chromosphere of magnetic regions that is related to the magnetic field strength observed in the lower layers at these locations on the disk.

Mechanisms for energy dissipation in the quiet Sun have been proposed including dissipation of strong acoustic shocks (e.g., Carlsson \& Stein 1992), magnetic field reconnection in nanoflares (e.g., Gallagher et al. 1999), and dissipation in small $(<160 \mathrm{~km})$ kilogauss structures of Pedersen current produced by convection (Goodman 2004). These mechanisms have not been quantitatively analyzed in models of the magnetic regions and it is yet unclear whether they would be able to explain the observations. Even if these mechanisms could be at work in solar magnetic regions it is not clear whether they are the main contributors to the observed heating.
Absorption of $\mathrm{p}$-mode motions propagating towards magnetic regions is well observed (Braun et al. 1990). Conversion to slow-mode waves is a viable alternative for explaining the p-mode absorption at magnetic regions (Cally et al. 2003) but it has not yet been proven to be the main process.

Wave motions propagating from high-beta regions towards magnetic regions with low-beta plasma would be in part reflected due to the increasing phase speed and in part converted into MHD waves. In particular, compression/rarefaction waves propagating upwards from sub-photospheric layers into active region areas with horizontal magnetic field would have essentially a p-mode character in low-beta photospheric layers but would convert to MHD fast-mode waves as they reach lower density layers where plasma-beta gradually becomes small. As magnetoacoustic waves propagate, electric fields are produced that drive charged particle drifts. The electric currents that result from these drifts have a very significant role in the wave propagation (contribute to the restoring force) and are limited by the collisions between charged and neutral particles, as well as by the interactions between charged particles. The magnetic field-induced particle gyration also affects these currents and this is usually described by a tensor electric conductivity that relates the electric field with the vector current (e.g., Saveliev \& Zheleznjakov 1991). The components of this tensor are named parallel, Pedersen, and Hall conductivity. Classical expressions are available for computing these components but, as we show, the classical collisional resistivity is insufficient for significant 
wave dissipation at frequencies not far from the measured in p-modes.

The well-known Farley-Buneman (FB) instability may arise due to the drift motions of magnetized particles. This instability has been associated with energy dissipation in the E-region of the ionosphere due to the small-scale electric fields the unstable modes produce. A theory for explaining the observed anomalous heating of electrons was developed by Hamza \& St. Maurice (1995). In this paper we study the parameters of the chromospheric plasma and the possibility of magnetoacoustic waves for driving the FB instability. This instability could allow the dissipation of magnetoacoustic wave energy and produce magnetic heating in the chromosphere.

In the FB instability we consider here, the neutral $\mathrm{H}$ atoms play an essential role since they strongly restrict the protons magnetization but allow that of electrons. Other plasma instabilities have been proposed for heating solar flare plasmas (e.g. Duijveman et al. 1981); however, these instabilities are different from the one we consider here.

\section{Basic plasma parameters}

We first examine the parameters of the plasma in typical magnetic regions of the solar atmosphere using the Fontenla et al. 2005 models. Each of these models corresponds to a typical configuration of the magnetic field. Here we will consider the normal network model $\mathrm{E}$, enhanced network model $\mathrm{F}$, plage model $\mathrm{H}$, and faculae model $\mathrm{P}$, which we associate with measured magnetic fields of about 10, 30, 100 and 300 Gauss, respectively. The magnetic field figures we use are typical observed values and we do not speculate about the possibility of unresolved magnetic structures with stronger fields. Also, for the purposes of this paper we assume a very simplified geometry in which the magnetic field is uniform, horizontal, and the direction of the $x$-axis. The atmospheric models resulted from observations characteristic in these categories of solar features but each category corresponds to a wide range of conditions. All physical quantities, e.g. temperature and densities, are assumed to vary only in the vertical direction, $z$-axis, corresponding to the 1-dimensional models. For the plots shown here we use the model $\mathrm{H}$ values but we have also graphed the parameters for all the models listed above and the results and considerations here apply to all these models. We show plots that contain the entire range from the photosphere to the transitionregions, but our focus is only the mid- and upper-chromosphere that show local magnetic heating and the other layers are just shown for reference to other conditions.

In the upper-chromospheric layers the electron and proton densities, $n_{\mathrm{e}}$ and $n_{\mathrm{p}}$, are very close and the heavy ions density, $n_{\mathrm{i}}$, is negligible for change balance. Also, according to the existing models, the ionized He density is several orders of magnitude smaller than the proton density and collisions of protons and electrons with neutral He have only a small effect because of the small cross-sections and He abundance $(0.1$ relative to $\mathrm{H})$. However, in the low chromosphere the total collision rates of electrons and protons are strongly increased by the Coulomb interaction with heavy ions. In the chromospheric layers close to the models temperature minimum, height $\sim 500 \mathrm{~km}$ above

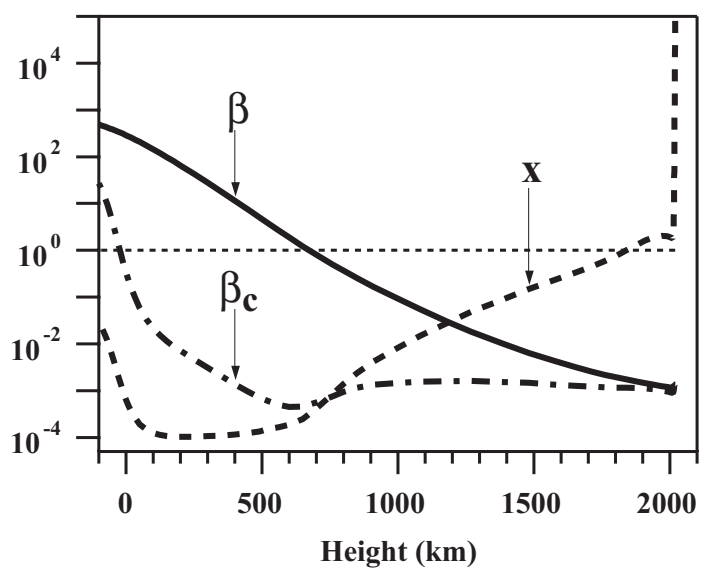

Fig. 1. Plasma beta, $\beta$, charged particles plasma beta, $\beta_{\mathrm{c}}$, and ionization ratio, $x=n_{\mathrm{e}} / n_{\mathrm{a}}$, as functions of height for model $\mathrm{H}$.

that at which $\tau_{5000}=1$, the combined heavy ions electric charge is about $10^{-4}$ times the total $\mathrm{H}$ density, $n_{\mathrm{H}}$, but more than an order of magnitude above the proton density. We compute the effects of the heavy ions approximately by assuming a heavy ionic species with $n_{\mathrm{i}}=10^{-4} n_{\mathrm{H}}$, unit charge, and an effective mass of 42 . The $\mathrm{H}$ ionization changes with height as the neutral $\mathrm{H}$ density, $n_{\mathrm{a}}$, decreases with an exponential-like shape while the proton density increases in the low chromosphere above the temperature minimum region and then decreases very slowly with height in the upper chromosphere.

As a result of these variations, the neutrals density is much larger than the proton and heavy ions densities at low heights (where heavy ions dominate the positive charge) and both densities become about equal near the top of the chromosphere (where protons dominate the positive charge). The ionization ratio, $x=n_{\mathrm{e}} / n_{\mathrm{a}}=\left(n_{\mathrm{p}}+n_{\mathrm{i}}\right) / n_{\mathrm{a}}$, and plasma beta, $\beta=p_{\text {gas }} / p_{\text {mag }}$ are displayed in Fig. 1.

This figure shows that in the upper-chromosphere the plasma beta is well below unity and the transition from above to below unity occurs at $\sim 600 \mathrm{~km}$ for model $\mathrm{H}$. For the other models the transition occurs at similar heights but slightly lower altitude $(\sim 400 \mathrm{~km})$ for model $\mathrm{P}$ and higher altitude $(\sim 1000 \mathrm{~km})$ for model $\mathrm{F}$. In model $\mathrm{E}$, the crossing occurs very high at $\sim 1400 \mathrm{~km}$.

The evolution of the plasma beta indicates that an upward propagating magnetoacoustic wave, in the geometry we specified, would have mostly acoustic behavior at photospheric layers where plasma beta is large. At upper chromospheric layers the magnetic component would dominate and the wave phase velocity would be large. An intermediate situation occurs at the low chromosphere. However, the fast-mode wave propagation is affected by the changing Alfven and sound speed velocity with height. Also shown in Fig. 1, the charged particle plasma beta, $\beta_{\mathrm{c}}=k T\left(n_{\mathrm{e}}+n_{\mathrm{p}}+n_{\mathrm{i}}\right) / p_{\text {mag }}$, is always small above the photosphere. In deep photospheric layers the charged particle plasma beta always increases and in the particular case of model $\mathrm{H}$ it may reach values above unity at heights below zero height (corresponding to $\tau_{5000}=1$ ).

Figure 2 shows the plasma frequencies for electrons and protons, $\omega_{\mathrm{e}}$ and $\omega_{\mathrm{p}}$, and the total momentum-exchange 


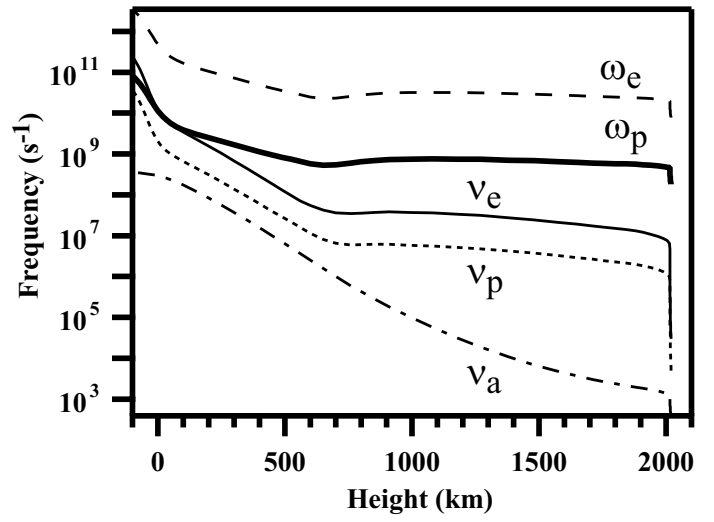

Fig. 2. Plasma $\omega$, and collision, $v$, frequencies as a function of height.

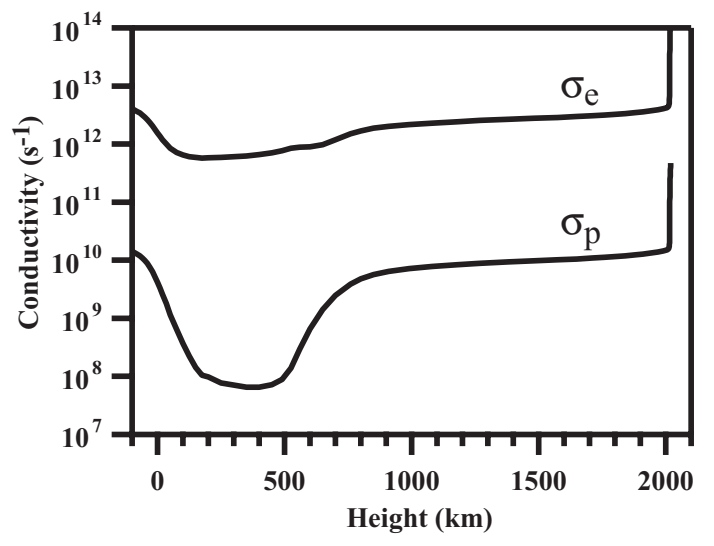

Fig. 3. Parallel electric conductivity, $\sigma$, a function of height.

collision rates for electrons, $v_{\mathrm{e}}$, protons $v_{\mathrm{p}}$, and neutral $\mathrm{H}$, $v_{\mathrm{a}}$, with respect to all other particles. This figure shows that charged particle collision rates are much smaller than the plasma frequency but much larger than those of neutral $\mathrm{H}$. The collision rates of electrons with neutral $\mathrm{H}, v_{\mathrm{ea}}$, is smaller than that with other charged particles. As a result of this, the parallel electrical conductivity is expected to be near the classical Spitzer 1962 value.

The proton-neutral $\mathrm{H}$ collisions are dominated by the charge exchange process that has a large cross-section at low collision energies and decreases rapidly with increasing collision energy (we estimated this collision rate following Fontenla et al. 1993). As usual the collisions between charged particles are dominated by the large frequency of relatively small deflections as usually described by Focker-Planck interaction (Landau 1936). Inelastic collisions, e.g. electron-proton recombination, occur at a much smaller rate. The electron-neutral $\mathrm{H}$ collisions can be elastic by interaction of the electron with the induced dipole, or inelastic leading to atom excitation/deexcitation or ionization/recombination but with a much smaller rate. We estimate the total collision cross-section for a collision of electrons with neutral $\mathrm{H}$ as about $\sigma_{\text {ea }}=10^{-15} \mathrm{~cm}^{2}$.

Figure 3 shows the electron and proton classical parallel conductivities as functions of height.

We compute the Larmour frequencies (or particle gyrofrequencies) for electrons and protons, $\Omega_{\mathrm{e}}$ and $\Omega_{\mathrm{p}}$ in the magnetic field. These frequencies are much smaller than the

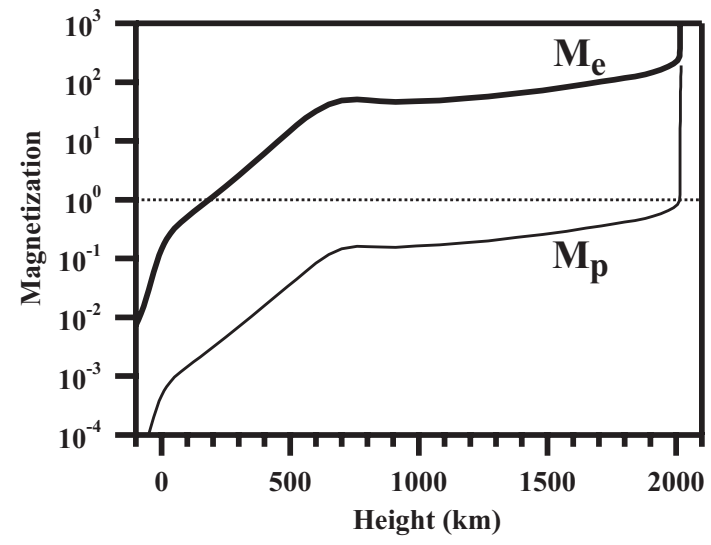

Fig. 4. Particle magnetization, $M$, as a function of height.

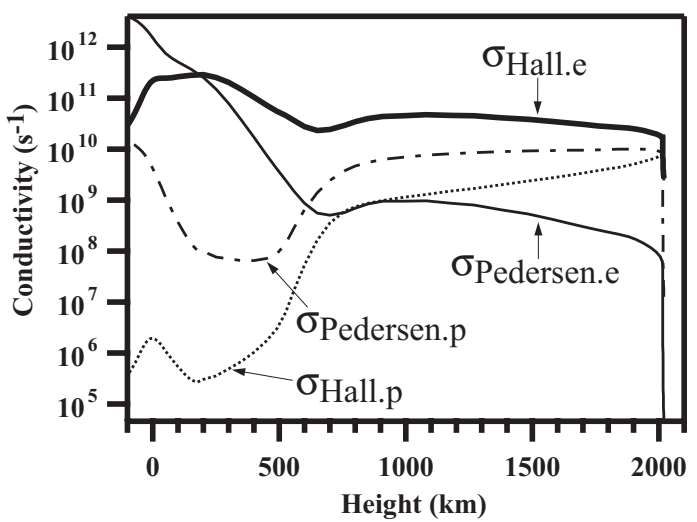

Fig. 5. Tensor electrical conductivity components as a function of height, in the absence of anomalous processes.

plasma frequency. Figure 4 shows the particle magnetizations, $M_{\mathrm{e}}=\Omega_{\mathrm{e}} / v_{\mathrm{e}}$ and $M_{\mathrm{e}}=\Omega_{\mathrm{p}} / v_{\mathrm{p}}$, that correspond to the ratio of the Larmour frequencies to the collision frequencies in Fig. 2. This figure shows that electrons are magnetized throughout the mid- and upper-chromosphere while the protons are unmagnetized (exchange momentum faster than they gyrate) up to the transition region.

Applying the usual formulas for the tensor electrical conductivity in low ionization plasma,

$\sigma_{\text {Pedersen.e }}=\frac{v_{\mathrm{ea}}^{2}}{v_{\mathrm{ea}}^{2}+\Omega_{\mathrm{e}}^{2}} \sigma_{\| \mathrm{e}}$,

$\sigma_{\text {Hall.e }}=\frac{v_{\mathrm{ea}} \Omega_{\mathrm{e}}}{v_{\mathrm{ea}}^{2}+\Omega_{\mathrm{e}}^{2}} \sigma_{\| \mathrm{e}}$

and the corresponding for protons, we find that the total Pedersen conductivity is dominated by protons and about the same as its parallel value. The total Hall conductivity has a large value and is dominated by electrons.

\section{MHD waves and particle drift and the Farley-Buneman instability threshold}

Let us consider fast-mode waves propagating upwards from the sub-photospheric layers into the chromosphere. At the peak of the wave the plasma moves upwards with a cross-field velocity 
amplitude $U$, and the usual center-of-mass electric $E=U \times B$ field arises in the direction of the $y$-axis. Electrons drift in the opposite direction of the electric field, and also in the direction of $z$ perpendicular to it. Protons drift in the direction of the electric field but not in the direction of $z$ because their frequent collisions with neutrals prevents them from gyration. Thus, a net drift of electrons with respect to protons would occur along the $z$-direction (wich is reflected in the Hall current).

The relative drift between electrons and protons gives rise to electric currents and is described by the electrical conductivity tensor. In fully-ionized plasmas there is a common drift of both electrons and protons along the $\mathrm{z}$-axis but this is not the case in the chromosphere due to the high neutral $\mathrm{H}$ density. As usual the Lorentz force due to the Pedersen electric current, $J_{\text {Pedersen }}$, and the background magnetic field produce part of the restoring force in MHD fast-mode waves, and this electric current produces energy dissipation at the rate $P=$ $J_{\text {Pedersen }} \cdot E=\sigma_{\text {Pedersen }} E^{2}$. Using the tensor conductivity values we computed for the mid- and upper-chromosphere we find that the magnetic diffusion coefficient, $D_{m}=c^{2} /\left(4 \pi \sigma_{\text {Pedersen }}\right)$, is small $\left(\sim 10^{11} \mathrm{~cm}^{2} \mathrm{~s}^{-1}\right)$ despite its increase due to the electrons magnetization. Considering wavelengths of $\sim 10^{7} \mathrm{~cm}$ or longer and frequencies of several $\mathrm{mHz}$, classical resistivity is too small to provide important wave dissipation.

In the situation we described it is possibile that the FarleyBuneman instability due to the relative drift of electrons with respect to protons excites electrostatic waves. This instability has been associated with anomalous phenomena observed in the Earth ionosphere E-region in which the similar case of partial ionization, magnetized electrons, and unmagnetized ions holds. The Farley-Buneman instability threshold is given by the condition (Milikh \& Dimant 2002; and Dimant \& Milikh 2003)

$E_{\mathrm{thr}}=B_{0} C_{\mathrm{s}}\left(1+\psi_{\perp}\right)$

or equivalently in our case by

$U_{\mathrm{thr}}=C_{\mathrm{s}}\left(1+\psi_{\perp}\right)$

where $E_{\text {thr }}$ is the threshold electric field, $U_{\text {thr }}$ is the threshold plasma velocity, $C_{\mathrm{s}}$ is the ion-acoustic speed, and

$C_{\mathrm{s}}=\sqrt{\frac{k\left(T_{\mathrm{e}}+T_{\mathrm{p}}\right)}{m_{\mathrm{p}}}}$

$\psi_{\perp}=\frac{v_{\mathrm{ea}} \nu_{\mathrm{pa}}}{\Omega_{\mathrm{e}} \Omega_{\mathrm{p}}}$

with $T_{\mathrm{e}}$ and $T_{\mathrm{p}}$ the electron and proton temperatures, respectively, and $m_{\mathrm{p}}$ the proton mass.

If we assume the electron and proton temperatures to be identical, as in the Fontenla et al. (2005) models, the ionacoustic speed is equal to the protons thermal speed given by the temperature specified in the model. Figure 6 shows the FB threshold term $\psi_{\perp}$ as a function of height in model $\mathrm{H}$.

As Fig. 6 shows the Farley-Buneman instability would be triggered in the mid- and upper-chromosphere by wave velocity

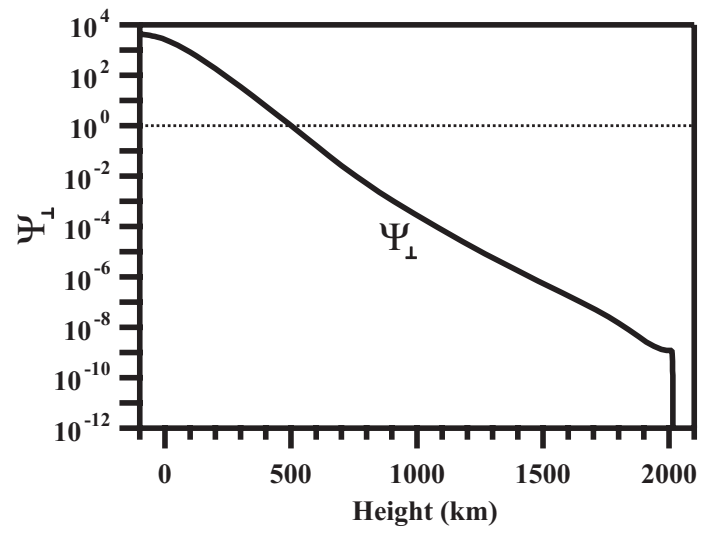

Fig. 6. Farley-Buneman threshold term $\psi_{\perp}$.

amplitudes larger or equal to the ion-acoustic speed, which is below but close to the adiabatic sound speed. At lower heights the threshold velocity amplitude increases rapidly requiring very supersonic velocity amplitudes for triggering the instability. Plots of this quantity for all models display a similar behavior but the height at which $\psi_{\perp}$ crosses unity is a bit higher, at $\sim 800 \mathrm{~km}$, for model E and a bit lower, at $\sim 400 \mathrm{~km}$, for model P.

The observed large width of the spectral lines with respect to the expected from the excitation-inferred electron temperature can be explained by the combination of the near-sonic mass motions in this layer and turbulent motions produced by the FB waves. High temporal- and spatial-resolution studies of line shapes and shifts would shed more light into this matter.

\section{Conclusions}

The triggering of any of plasma instabilities has important consequences on the MHD wave propagation because it would produce anomalous resistivity and energy dissipation. An increase in the Pedersen resistivity of about 2 orders of magnitude over its classical value would be sufficient to produce very strong MHD wave dissipation and heating. Besides, an increased resistivity would also allow dissipation of other electric currents that are not directly related to the MHD waves.

The conditions for the FB instability would result from upward-propagating p-modes as the velocity amplitude increase with height because of the rapidly-decreasing density of neutrals. The amplitudes of $\mathrm{p}$-mode waves in the absence of magnetic fields are expected to reach values close to the sound speed in the low-chromosphere and to become supersonic in the upper-chromosphere (Carlsson \& Stein 1992). The shocks resulting from this have been suggested to produce heating in un-magnetized regions. However, in the presence of horizontal magnetic fields the p-mode waves would convert to fast-mode MHD modes with an increasing magnetic component as the plasma beta decreases. As the wave velocity amplitude reaches the threshold for the FB instability part of the wave magnetic component would dissipate. This process decreases the wave energy reflection that would otherwise occur due to the increasing wave phase velocity (Stark \& Musielak 1993). Also, the wave energy dissipation would produce heating at a frequency double of that of the wave and given the relatively long cooling 
time of the chromosphere it would result in an almost steady increased temperature but with some fluctuation. The result is very different from the effects of non-magnetic acoustic shocks.

In a quick analysis, as the anomalous resistivity decreases the MHD fast-mode restoring force, the velocity $U$ would increase leading to a larger wave electric field and energy dissipation (that depends on the square of this field). This could create a runaway process by which at the instability saturation the anomalous resistivity would dissipate the magnetic part of the wave energy flow. Since the wave magnetic part dominates in the upper chromosphere, most of the wave energy would be absorbed throughout the chromosphere, thus explaining the observed p-mode absorption in magnetic regions. Also, the wave propagation and reflection would be strongly affected by this dissipation in the region where the plasma beta is smaller than unity and less affected otherwise. This could produce interesting observable effects on the part of the wave energy that is still reflected. The FB instability growth rate is smaller than the electron plasma frequency but still very fast compared to the MHD wave frequencies we consider. Also, the collision frequencies are very large and therefore the wave electric field and current are practically DC from the point of view of the FB instability growth and saturation. Consequently, the associated anomalous resistivity would develop quickly and almost identically for both the compression and rarefaction half-cycles of the MHD wave leading to energy dissipation increasing with the square of the instantaneous wave velocity. The overall effects of this process on the MHD wave propagation and absorption are non-linear and complicated; they need to be further studied.

Once developed, anomalous resistivity can also help dissipate parallel electric currents built by other processes. Another interesting consideration is that the charge exchange process that dominates the proton-neutral collision does not have a random effect the velocity distributions of the involved particles but instead it exchanges the proton and neutral $\mathrm{H}$ velocities. The implications of this are complicated and under study.

MHD wave dissipation through FB instability can explain both sustained heating in magnetic regions and p-mode absorption. In this picture both phenomena would be closely related and strongly dependent on the background magnetic field. When the field is too small, or the neutral $\mathrm{H}$ density too high, the conditions for this mechanism to operate would disappear at some layers. However, since the neutral $\mathrm{H}$ density decreases with height at a rate much faster than the proton density, the proper conditions to excite the FB instability would occur at some higher altitude even in a small background magnetic field. The mechanism we propose requires a propagating wave whose velocity amplitude increases with height and reaches near the sound speed. Our study indicates that the conditions for instability are met in decreasing altitude chromospheric layers as the field increases and this is coarsely consistent with the observations but more detailed studies of the existing observation are needed.

The current atmospheric models assume the height of the start of the chromospheric temperature rise at a fixed altitude independent of the magnetic field and this may not be realistic. Also, the current models arbitrarily assume equal electron and heavy particle temperatures and this also may be unrealistic in the scenario we propose. The considerations from this paper point to a number of studies that could improve our understanding of the chromosphere. One of these is to explore further the possibility of important differences between the electron and heavy particles temperature and how this could be reflected in observations of the spectrum.

An important step to validate the scenario proposed here is to check for observable signatures of this process. For instance, the velocity distribution of the neutral $\mathrm{H}$ can be inferred from the Lyman line profiles other than $\operatorname{Ly} \alpha$, or in other lines, and may reflect non-isotropic characteristics correlated with particular orientations of the magnetic fields and with wave phase. Also electron temperature and turbulence increase would be a function of the absolute value of the instantaneous velocity. However, the analysis of the observations is involved because all chromospheric lines are optically thick.

Another test for this scenario is to develop the equations further and perform forward simulations so that one can evaluate quantitatively the anomalous resistivity and the amount of energy dissipation and use these in simulations of the MHD wave propagation. These simulations would indicate which detailed observations would be expected.

Acknowledgements. This work was supported by NASA contract NAS5-97045 at the University of Colorado.

\section{References}

Braun, D. C., Labonte, B. J, \& Duvall, T. L. Jr 1990, ApJ, 354,372

Cally, P. S., Crouch, A. D., \& Braun, D. C. 2003, MNRAS, 346, 381

Carlsson, M., \& Stein, R. F. 1992, ApJ, 397, L59

Dimant, Y. S., \& Milikh, G. M. 2003, GRL, 108, 1350

Duijveman, A., Hoyng, P., \& Ionson, J. A. 1981, ApJ, 245, 721

Fontenla, J. M., Avrett, E. H., \& Loeser, R. 1993, ApJ, 406, 319

Fontenla, J. M., Avrett, E. H., Thuillier, G., \& Harder, J. 2005, ApJ, submitted

Gallagher, P. T., Phillips, K. J. H., Harra-Murnion, L. K., Baudin, F., \& Keenan, F. P. 1999, A\&A, 348, 251

Goodman, M. L 2004, A\&A, 416, 1159

Hamza, A. M., \& St. Maurice, J.-P. 1995, JGR, 100, 9653

Landau, L. 1936, Phys. Z. Sowj. Un., 10, 154

Lemaire, P., Gouttebroze, P., Vial, J.-C., \& Artzener, G. 1981, A\&A, 103,160

Milikh, G. M., \& Dimant, Y. S. 2002, GRL, 29, 1575

Saveliev, V. L., \& Zheleznjakov, E. V. 1991, P\&SS, 39, 1133

Spitzer, L. 1962, Physics of Ionized Gases (New York: Interscience)

Stark, B. A., \& Musielak, Z. E. 1993, ApJ, 4090, 450 